\title{
$A B C B 1$ SNP predicts outcome in patients with acute myeloid leukemia treated with Gemtuzumab ozogamicin: a report from Children's Oncology Group AAML0531 Trial
}

Roya Rafiee ${ }^{1}$, Lata Chauhan' ${ }^{1}$, Todd A. Alonzo ${ }^{2}$, Yi-Cheng Wang ${ }^{3}$, Ahlam Elmasry ${ }^{4}$, Michael R. Loken ${ }^{5}$, Jessica Pollard ${ }^{6,7}$, Richard Aplenc ${ }^{8}$, Susana Raimondi ${ }^{9}$, Betsy A. Hirsch ${ }^{10}$, Irwin D. Bernstein ${ }^{11}$, Alan S. Gamis ${ }^{12}$, Soheil Meshinchi ${ }^{11}$ and Jatinder K. Lamba (iD ${ }^{1}$

\begin{abstract}
Gemtuzumab-ozogamicin (GO), a humanized-anti-CD33 antibody linked with the toxin-calicheamicin- $\gamma$ is a reemerging and promising drug for AML. Calicheamicin a key element of GO, induces DNA-damage and cell-death once the linked CD33-antibody facilitates its uptake. Calicheamicin efflux by the drug-transporter PgP-1 have been implicated in $\mathrm{GO}$ response thus in this study, we evaluated impact of ABCB1-SNPs on GO response. Genomic-DNA samples from 942 patients randomized to receive standard therapy with or without addition of GO (COG-AAML0531) were genotyped for ABCB1-SNPs. Our most interesting results show that for rs 1045642, patients with minor-T-allele $(C T / T T)$ had better outcome as compared to patients with CC genotype in GO-arm (Event-free survival-EFS: $p=0.022$; and risk of relapse-RR, $p=0.007$ ). In contrast, no difference between genotypes was observed for any of the clinical endpoints within No-GO arm (all $p>0.05$ ). Consistent results were obtained when genotype groups were compared by GO and No-GO arms. The in vitro evaluation using HL60-cells further demonstrated consistent impact of rs 1045642T-allele on calicheamicin induced DNA-damage and cell-viability. Our results show the significance of ABCB1 SNPs on $\mathrm{GO}$ response in $\mathrm{AML}$ and warrants the need to investigate this in other cohorts. Once validated, ABCB1-SNPs in conjunction with CD33-SNPs can open up opportunities to personalize GO-therapy.
\end{abstract}

\section{Introduction}

Gemtuzumab ozogamicin (GO), a CD33-antibody conjugated to cytotoxin-calicheamcin ${ }^{1}$ is a re-emerging and promising drug in AML treatment. In light of encouraging results from several randomized studies $^{2-8}$,

\footnotetext{
Correspondence: Jatinder K. Lamba (jlamba@cop.ufl.edu)

${ }^{1}$ Department of Pharmacotherapy and Translational Research, Center for

Pharmacogenomics, College of Pharmacy, University of Florida, Gainesville, FL,

USA

${ }^{2}$ Keck School of Medicine, University of Southern California, Los Angeles, CA,

USA

Full list of author information is available at the end of the article.

Previous presentation: This work has been presented at the Annual American

Society of Hematology meeting in Atlanta, 2017.

These authors contributed equally: Roya Rafiee, Lata Chauhan.
} 
associated with in vitro GO-sensitivity and clinical response in AML patients ${ }^{10-14}$. In vitro cellular sensitivity of unconjugated-calicheamicin varies >100,000-fold between the most-resistant and most-sensitive AML ${ }^{15}$. Unfortunately, the impact of genetic-polymorphisms in $A B C B 1$ on the clinical-efficacy of $\mathrm{GO}$ has never been investigated. In this study, we evaluated $A B C B 1$ SNPs in pediatric AML patients randomized to the addition of two doses of GO to standard chemotherapy ${ }^{8}$.

\section{Materials and methods}

\section{Patients and treatment}

This study included specimens from pediatric de novo AML patients (ages 0-29 years) randomized to receive standard five-course chemotherapy (No-GO arm, $n=$ 511 ) or the same chemotherapy with addition of two doses of $3 \mathrm{mg} / \mathrm{m}^{2}$ GO (GO arm, $n=511$ ) during induction I and intensification II in COG-AAML0531 trial. The study design, treatment regimen and clinical outcome details have been reported previously ${ }^{8}$. Low-risk group included presence of $t(8 ; 21)$, inv(16), or $t(16 ; 16)$. High-risk group included monosomy 7 , monosomy $5 / 5$ q deletion, or persistent disease at end of induction 1 and all high-risk group patients received allogeneic stem cell transplant. Patients with absence of low or high-risk features were assigned intermediate-risk group and received HSCT if suitable donor was available ${ }^{8,16}$. Specimens from patients who consented for biology studies were used in this study. The institutional review boards of all participating institutions approved the clinical protocol, and the COGMyeloid Disease Biology Committee approved this research.

\section{Genotyping}

In this study, we genotyped $12 A B C B 1$ SNPs in genomic DNA from 942 children and young adults enrolled on COG-AAML0531. Genotyping was performed using the Sequenome platform at the Biomedical Genomics Center, University of Minnesota. Three SNPs with minimum allele frequency of $<0.1$ or call-rate of $<0.95$ were excluded (Supplementary Table 1). Of the 9 SNPs that were included in the study 3 SNPs (rs1045642; rs1128503; and rs2032582) occur in partial LD with each other.

\section{Statistical analysis}

Statistical analysis was performed to compare clinical endpoints: overall-survival (OS), and event-free-survival (EFS) from study entry, disease-free-survival (DFS) from end of Course 1 (EOC1) and risk of relapse (RR) from EOC1. Analysis was performed: (i) between genotype groups within each arm and (ii) between GO and No-GO arms within different genotype groups in all patients as well as within risk-groups ${ }^{8}$ using Cox regression and Fisher's exact test.

\section{In vitro evaluation of $A B C B 1$ SNP-rs 1045642}

HL-60 cells, a promyelocytic cell line with low näive expression of ABCB1 (ATCC, Manassas, VA, USA) was select to evaluate ABCB1 SNP -rs1045642. Cell line was cultured in medium containing 10\% FBS and 1\% Glutamine (Invitrogen, USA) at $37^{\circ} \mathrm{C}$ in humidified incubator containing $5 \% \mathrm{CO}_{2}$ as per manufacturer's instructions.

Cells were transfected with either control -pCl-neo or $A B C B 1$ expression vectors: ABCB1- rs1045642-C, and rs1045642-T using nucleofector2b (Lonza, Switzerland) as per manufacture's protocol. Forty-eight hours posttransfection cell surface expression levels of Pgp-1 was analyzed using $\mathrm{ABCB} 1 \mathrm{mAB}-\mathrm{UIC} 2$ conjugated with Phycoerythrin (PE), (Abcam, ab93590) by BDTM FACS LSR II. $48 \mathrm{~h}$ post-transfection cells were exposed to $40 \mathrm{nM}$ calicheamicin for $24 \mathrm{~h}$ and cell viability evaluated using NucRed Live (Invitrogen, USA) and AO-PI (Acridine orange and propidium iodide) staining.

We also evaluated calicheamicin induced DNA-damage in cells transfected with control and ABCB1-expression vectors using comet assays. Post-transfection, cells were treated with $40 \mathrm{nM}$ calicheamicin for $45 \mathrm{~min}$ followed by immobilization in a bed of low (1\%) melting agarose. Cells were treated with alkali to unwind the DNA. Electrophoresis and subsequent staining with SYBER gold nucleic acid gel stain (Invitrogen, USA) was performed and percent of DNA tail-length was estimated using fluorescence microscopy and image J software.

\section{Results}

Clinical response within $\mathrm{GO}$ and No-GO arms by $A B C B 1$ SNP genotypes

Initial analysis evaluated association of $A B C B 1$ SNPs with clinical outcome within GO arm or No-GO arm. rs1045642 C>T, which is one of the most studied SNP within $A B C B 1$ showed significant association of genotype with response. Patients with minor allele (CT/TT) had a significantly better outcome with $\mathrm{GO}$ as compared to patients with $\mathrm{CC}$ genotype (GO arm: 5 years EFS: $\mathrm{CC}=$ $44 \pm 9 \%, \mathrm{CT}=55 \pm 7 \%, \mathrm{TT}=56 \pm 10 \%, \mathrm{CC}$ vs. $\mathrm{CT} / \mathrm{TT}$ $p=0.022$; DFS, CC $=51 \pm 10 \%, \mathrm{CT}=62 \pm 7 \%$, TT $=64 \pm$ $11 \%, \mathrm{CC}$ vs. CT/TT $p=0.044$ and $\mathrm{RR}, \mathrm{CC}=45 \pm 10 \%$, $\mathrm{CT}=30 \pm 7 \% \mathrm{TT}=28 \pm 10 \%$, CC vs. CT/TT $p=0.007$, Table 1; Fig. 1a). In contrast, no difference between genotypes was observed for any of the clinical endpoints within standard No-GO arm (all $p>0.05$ ). Given that riskgroups impact clinical responses to $\mathrm{GO}$ and allele-frequency of rs1045642 SNP differ by risk-group classification (Supplementary Table 2), we evaluated $A B C B 1$ SNPs within low, standard and high-risk group patients by treatment arms. As shown in Fig. 1b, we observed significant association of rs1045642 SNP with outcome in $\mathrm{GO}$ arm within only standard-risk group patients (GO arm: EFS, $p=0.014$, DFS, $p=0.006$ and RR, 


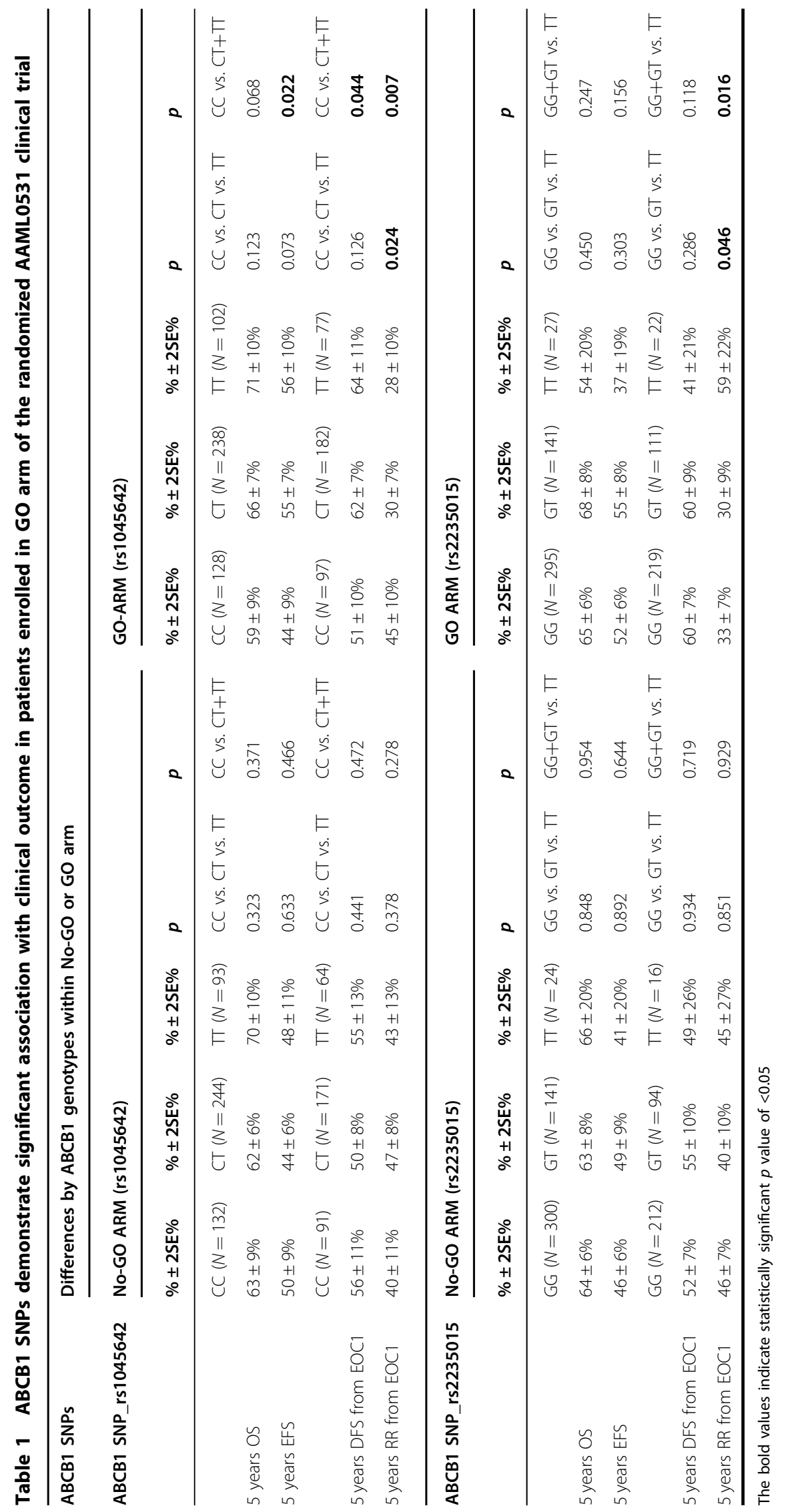




\section{A) rs1045642 genotypes within GO or NO-GO treatment arms in all Patients}
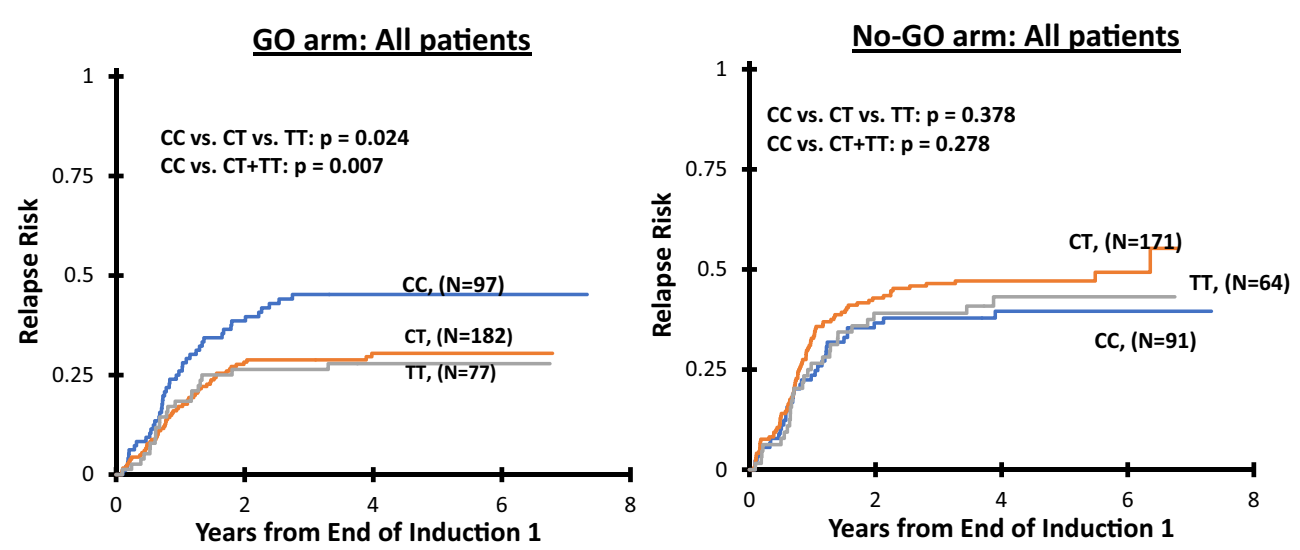

B) rs1045642 genotypes within GO or NO-GO treatment arms in Standard Risk Group Patients
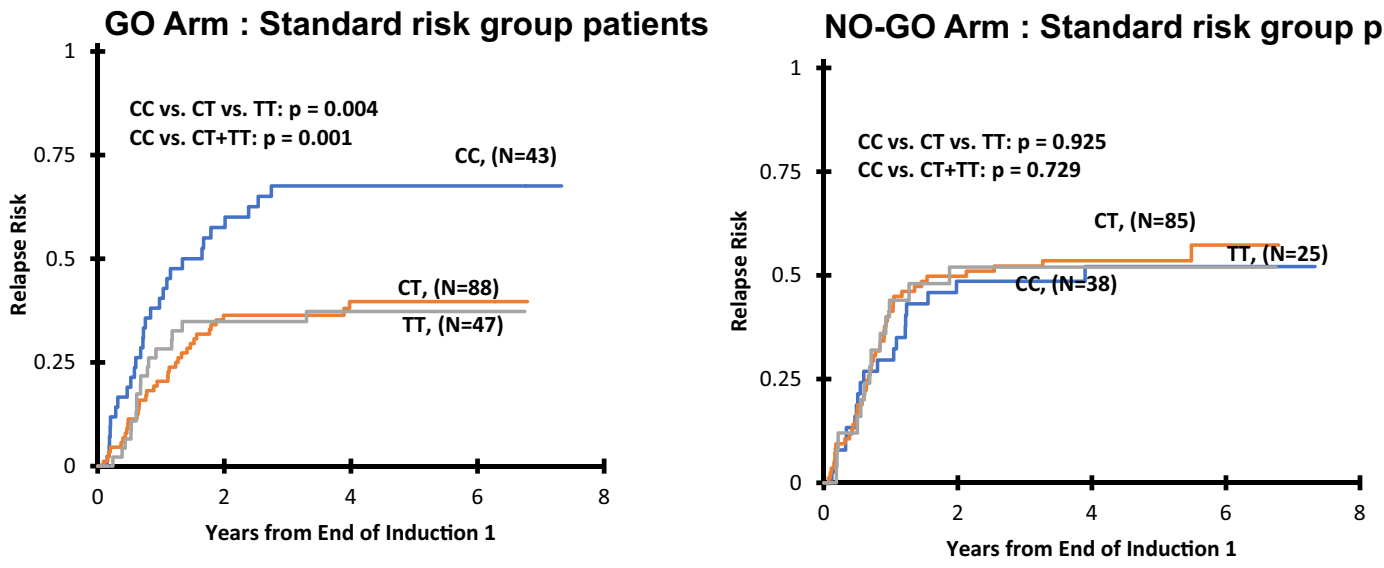

Fig. 1 Association of $A B C B 1$ SNP rs 1045642 with clinical outcome in patients from AAML0531 trial. Comparison of risk of relapse from end of course 1 by rs 1045642 genotype within GO or No-GO arms in all patients (a) and within standard risk group patients (b)

$p=0.004$ for CC vs. CT vs. TT genotypes, Fig. 1b; Supplementary Table 3).

For a less frequent intronic-SNP rs2235015-G>T that occurs with the allele frequency of $(0.207)$ presence of $G$ allele (GG/GT genotypes) was associated with lower RR (GG/GT vs. TT, $p=0.016$ ) only in GO arm, whereas no association between genotypes and outcome was observed in No-GO arm $(p>0.05$, Table 1$)$.

\section{Clinical response within $A B C B 1$ SNP genotypes by $\mathrm{GO}$ and No-GO arms}

Second analysis evaluated difference in clinical outcome comparing No-GO and GO treatment arms within different genotype groups for $A B C B 1$ SNPs and showed consistent with results as observed in within arm analysis above. Patients with rs1045642-CT/TT genotype had better EFS (No-GO vs. GO: $45 \pm 5 \%$ vs. $55 \pm 6 \%, p=0.005$ ), DFS (No-GO vs. GO: $51 \pm 5 \%$ vs. $62 \pm 6 \%, p=0.008$ ) and RR
(No-GO vs. GO: $46 \pm 7 \%$ vs. $30 \pm 6 \%, p<0.001$; Fig. 2; Table 2) when treated on GO containing arm as compared to No-GO-arm. In contrast no difference in outcome between GO vs. No-GO arms was observed for patients within rs1045642-CC genotype group (all $p>0.05$ ).

This association of improved clinical outcome within patients with at least one $\mathrm{T}$ allele in response to GO addition was consistent across the three risk group categories. Within standard-risk group patients, presence of rs1045642-T allele was associated with improved outcome in GO recipients only (No-GO vs. GO; EFS: $37 \pm 7 \%$ vs. $49 \pm 8 \%, p=0.006$, DFS: $45 \pm 10 \%$ vs. $57 \pm 9 \%, p=$ 0.024 and RR: $53 \pm 10 \%$ vs. $39 \pm 9 \%, p=0.008$, Supplementary Table 4; Fig. 2b). Within low and high-risk group presence of $\mathrm{T}$ allele (CT/TT) was associated with lower RR (No-GO vs. GO; low-risk group RR: $35 \pm 10 \%$ vs. $16 \pm$ $8 \%, p=0.005$ and high-risk group RR: $64 \pm 21 \%$ vs. $28 \pm$ $18 \%, p=0.018$ ) (Supplementary Table 4). In contrast for 


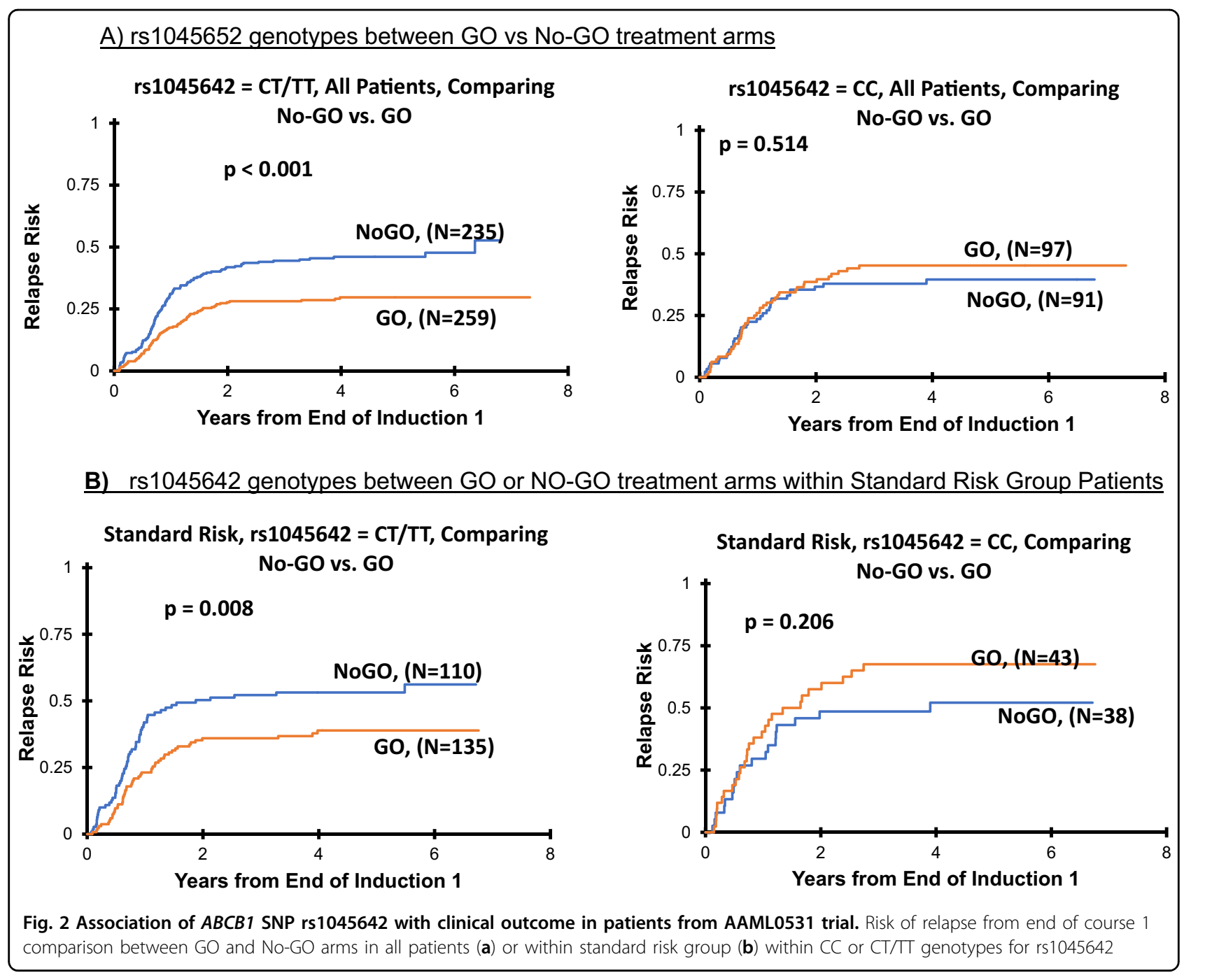

rs1045642-CC genotype, no difference in outcome within risk groups was observed in GO vs. No-GO arms (all $p>$ 0.05). These results were consistent within genotype groups for the SNPs rs1128503 and rs2032582, which occurs in partial LD with rs1045642 (Supplementary Table 5) as well as for the intronic SNP-rs2235015 (Table 2).

A multivariate Cox regression analysis that included rs1045642, risk status, and CD33 expression (CD33 expression has been shown to impact GO response ${ }^{16,17}$ ) demonstrated that treatment-arm is significant predictor of outcome for rs1045642 CT/TT genotype (Hazard Ratio $(\mathrm{HR})=0.543$ and $p<0.001$ for DFS; HR $=0.428$ and $p<$ 0.001 for $R R$ ) similar results were observed for the rs2235015 GG/GT genotype (HR $=0.66$ and $p=0.004$ for DFS; $H R=0.56$ and $p<0.001$ for RR; Supplementary Table 6).
Interaction between ABCB1 rs1045642 and CD33 splicing SNP rs12459419 on clinical outcome

We recently reported a splicing SNP-rs12459419 C>T in CD33, that results in loss of IgV domain (which is recognized by $\mathrm{GO}$ ) to be significant predictor of clinical outcome in response to $\mathrm{GO}^{9}$. For this splicing SNP-CC genotype had significant benefit with almost $50 \%$ reduction of risk of relapse in GO arm as compared to standard No-GO arm, whereas no benefit of adding GO is seen in $\mathrm{CT} / \mathrm{TT}$ genotype. In light of these results. we evaluated $A B C B 1-\mathrm{rs} 1045642$ and CD33-rs12459419 SNP-SNP interaction within COG-AAML0531-GO arm. Within patients with $C D 33$ rs12459419-CC genotype, $A B C B 1$ SNP was not associated with outcome (GO arm, RR, rs $1045642 \mathrm{CC}=35 \pm 13.9 \%, \mathrm{CT}=28 \pm 9.2 \%$, TT $=22.6 \pm$ $15.3 \%, p=0.594)$, however in CD33-rs12459419-CT genotype group, presence of $A B C B 1$-rs1045642-CC was 
Table 2 ABCB1 genotype demonstrates significant difference in clinical outcome in GO vs. No-GO arms in patients enrolled in randomized AAML0531 clinical trial

\begin{tabular}{|c|c|c|c|c|c|c|}
\hline \multirow{3}{*}{$\begin{array}{l}\text { ABCB1 SNPs } \\
\text { ABCB1 SNP_rs1045642 }\end{array}$} & \multicolumn{6}{|c|}{ Differences in No-GO vs GO treatment arms by $A B C B 1$ SNP genotypes } \\
\hline & \multicolumn{3}{|l|}{ CC $(N=260)$} & \multicolumn{3}{|l|}{$\mathrm{TT} / \mathrm{CT}(N=677)$} \\
\hline & $\% \pm 2 \mathrm{SE} \%$ & $\% \pm 2 S E \%$ & $p$ & $\% \pm 2 \mathrm{SE} \%$ & $\% \pm 2 S E \%$ & $p$ \\
\hline & No-GO $(N=132)$ & $\mathrm{GO}(N=128)$ & & No-GO $(N=337)$ & $\mathrm{GO}(N=340)$ & \\
\hline 5 years OS & $63 \pm 9 \%$ & $59 \pm 9 \%$ & 0.851 & $64 \pm 5 \%$ & $67 \pm 5 \%$ & 0.391 \\
\hline \multirow[t]{2}{*}{5 years EFS } & $50 \pm 9 \%$ & $44 \pm 9 \%$ & 0.425 & $45 \pm 5 \%$ & $55 \pm 6 \%$ & 0.005 \\
\hline & No-GO $(N=91)$ & $\mathrm{GO}(N=97)$ & & No-GO $(N=235)$ & $\mathrm{GO}(N=259)$ & \\
\hline 5 years DFS from EOC1 & $56 \pm 11 \%$ & $51 \pm 10 \%$ & 0.561 & $51 \pm 7 \%$ & $62 \pm 6 \%$ & 0.008 \\
\hline 5 years RR from EOC1 & $40 \pm 11 \%$ & $45 \pm 10 \%$ & 0.514 & $46 \pm 7 \%$ & $30 \pm 6 \%$ & $<0.001$ \\
\hline \multirow[t]{3}{*}{ AB CB1 SNP_rs2235015 } & \multicolumn{3}{|l|}{$\mathrm{TT}(N=51)$} & \multicolumn{3}{|l|}{ GG/GT $(N=877)$} \\
\hline & $\% \pm 2$ SE $\%$ & $\% \pm 2$ SE $\%$ & $p$ & $\% \pm 2$ SE $\%$ & $\% \pm 2 S E \%$ & $p$ \\
\hline & No-GO $(N=24)$ & $\mathrm{GO}(N=27)$ & & No-GO $(N=441)$ & $\mathrm{GO}(N=436)$ & \\
\hline 5 years OS & $66 \pm 20 \%$ & $54 \pm 20 \%$ & 0.579 & $64 \pm 5 \%$ & $66 \pm 5 \%$ & 0.449 \\
\hline \multirow[t]{2}{*}{5 years EFS } & $41 \pm 20 \%$ & $37 \pm 19 \%$ & 0.940 & $47 \pm 5 \%$ & $53 \pm 5 \%$ & 0.049 \\
\hline & No-GO $(N=16)$ & $\mathrm{GO}(N=22)$ & & No-GO $(N=306)$ & $\mathrm{GO}(N=330)$ & \\
\hline 5 years DFS from EOC1 & $49 \pm 26 \%$ & $41 \pm 21 \%$ & 0.903 & $53 \pm 6 \%$ & $60 \pm 6 \%$ & 0.036 \\
\hline 5 years RR from EOC1 & $45 \pm 27 \%$ & $59 \pm 22 \%$ & 0.585 & $44 \pm 6 \%$ & $32 \pm 5 \%$ & 0.001 \\
\hline
\end{tabular}

EOC1 end of course 1

The bold values indicate statistically significant $p$ value of $<0.05$

associated with inferior outcome as compared to $A B C B 1$ rs1045642 CT/TT genotypes (within rs12459419-CT RR: rs1045642- $\mathrm{CC}=59.7 \pm 16.3 \%, \mathrm{CT}=30.8 \pm 11.4 \%, \mathrm{TT}=$ $28.1 \pm 15.4 \%$, CC vs. CT vs. TT, $p=0.005$, CC vs. CT/TT $p=0.001$; DFS within rs12459419 CT: rs1045642- CC $=$ $35.2 \pm 15.5 \% \mathrm{CT}=65 \pm 11.6 \% \mathrm{TT}=62.4 \pm 16.7 \%, \mathrm{CC}$ vs. CT vs. TT, $p=0.005$, CC vs. CT/TT, $p=0.001$ ).

\section{In vitro evaluation of $A B C B 1$ rs10456C $>T$ SNP on calicheamicin response}

For in vitro evaluation, we selected HL-60 cell line with low naive expression of ABCB1. Cells were transfected with empty expression vector or expression vectors expressing ABCB1 WT (rs1045642 C) or ABCB1 -rs1045642 T variant. Cell surface Pgp-1 was determined $48 \mathrm{~h}$ post-transfection using UIC2 ABCB1 antibody conjugated with PE. The mean fluorescence intensity (MFI) analyzed on a 5 decade log scale $(1-100,000)$ confirmed over expression in transfected cells compare to naive cells. DNA damage was evaluated using comet assay that demonstrated significant increased in DNA damage in response to calicheamicin treatment ( $40 \mathrm{nM}$ for $0.75 \mathrm{~h}$ ) in HL60 expressing ABCB1-rs1045642T variant allele as compared to ABCB1-WT ( $p<0.05$; Fig. 3a). This implies reduced accumulation of calicheamicin as a potential reason for reduced DNA damage in cells expressing ABCB1-WT. Corresponding to this calicheamicin induced cell death was greater in cells expressing ABCB1C3435T as compared to the ABCB1-WT (Fig. 3b, $p<$ $0.05)$ expression constructs when tested using NucRed live and AOPI staining.

\section{Discussion}

$A B C B 1$ has been previously shown to impact response to gemtuzumab ozogamicin. In this study, we evaluated whether the most studied SNPs within $A B C B 1$ have an influence on treatment outcome in patients receiving $\mathrm{GO}$ based therapy in the randomized AAML0531 clinical trial. rs1045642, also commonly referred to as C3435T is a synonymous change (Ile1145Ile) in exon 27 of $A B C B 1$ and has been shown to occur in partial LD with two coding SNPs rs1128503 (Gly412Gly) and rs2032582 (Ser893Ala/ Thr). rs1045642-T allele has been shown to be associated with lower $A B C B 1$ expression in several studies ${ }^{18-22}$, and with higher expression in one study ${ }^{23}$. Hoffmeyer et al. linked TT genotype of rs1045642 with lower expression 
A) Calicheamicin induced DNA damage in HL60 cells transfected with $A B C B 1$ expression vectors

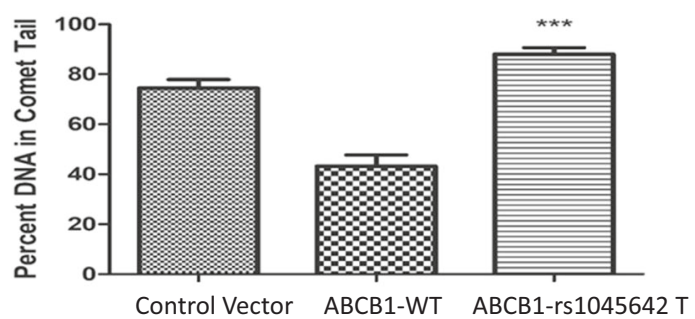

B) Calicheamicin induced cell death in HL60 cells transfected with $A B C B 1$ expression vectors

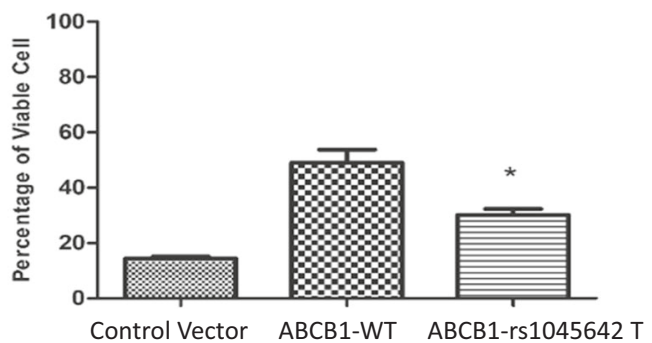

Fig. 3 a Comet Assay: percent tail DNA measured in HL60 cells transfected with ABCB1 expression constructs in response to calicheamicin treatment. Histograms represented fluoresce microscopy data were analyzed by Image J software to calculate average of tails per each group $\left({ }^{* * *} p<0.001\right)$. b Calicheamicin reduced cell viability in HL-60 cells expressing ABCB1-1045642T as compared to ABCB1-WT as determined by AOPI. Dual-

fluorescence viability, Acridine orange $(\mathrm{AO})$ and propidium iodide $(\mathrm{PI})\left({ }^{*} \mathrm{p}<0.05\right)$

and activity of $A B C B 1^{24}$. Although more work is required to establish the clear relationship between $A B C B 1$ genotypes and calicheamicin efflux, our results are the first to indicate that presence of $A B C B 1$ - low-expressing rs1045642 TT genotype might increase intracellular abundance of calicheamicin due to reduced efflux in leukemic cells, which in turn can enhance chemosensitivity to GO. Our results warrant further investigation of $A B C B 1$ SNPs in context of gemtuzumab response in additional clinical cohorts.

Interestingly, SNP-SNP interaction between $A B C B 1$ SNP and recently reported CD33 splicing SNP implies that within CD33-rs12459419-CT, $A B C B 1$ genotype is critical for calicheamicin abundance and thus might contribute towards poor response observed within rs1045642-CT group. Our cohort was limited by sample size to perform SNP-SNP interaction evaluation, warranting the need for validation in additional cohorts.

Our results also open up opportunities to further investigate and validate impact of $A B C B 1$ SNPs in other $\mathrm{GO}$ randomized clinical trials. Once validated, $A B C B 1$ SNPs in conjunction with CD33-SNPs can provide more accurate prediction of response to $\mathrm{GO}$ opening up opportunities to personalize GO-therapy.

\section{Acknowledgements}

This research was supported by $\mathrm{NIH}$ under the award numbers: U10CA180899, U10CA180886, U10CA98413, U10CA098543 as well as by NCI-R21CA155524, R01CA132946, and R01CA133881. The research was also supported by St. Baldrick's Foundation. Authors would like to thank the patients and families for participating in AAML0531. We are also thankful to Dr. Roland Walter for provide insightful comments. We are thankful to Drs Gottesman and Wang for providing $A B C B 1$ expression constructs.

\footnotetext{
Author details

${ }^{1}$ Department of Pharmacotherapy and Translational Research, Center for Pharmacogenomics, College of Pharmacy, University of Florida, Gainesville, FL, USA. ${ }^{2}$ Keck School of Medicine, University of Southern California, Los Angeles, CA, USA. ${ }^{3}$ Children's Oncology Group, Monrovia, CA, USA. ${ }^{4}$ Mansoura University, Mansoura, Egypt. ${ }^{5}$ Hematologics Inc, Seattle, USA. ${ }^{6}$ Dana-Farber/ Boston Children's Cancer Center and Blood Disorders Center, Boston, MA, USA. ${ }^{7}$ Harvard Medical School, Boston, MA, USA. ${ }^{8}$ Division of Oncology, Children's
}

Hospital of Philadelphia, Philadelphia, PA, USA. ${ }^{9}$ Department of Pathology, St. Jude Children's Hospital, Memphis, TN, USA. ${ }^{10}$ Children's Hospitals and Clinic of Minnesota, University of Minnesota, Minneapolis, MN, USA. ${ }^{11}$ Clinical Research Division, Fred Hutchinson Cancer Research Center, Seattle, WA, USA. ${ }^{12}$ Division of Hematology/Oncology/Bone Marrow Transplantation, Children's Mercy Hospitals and Clinics, Kansas City, MO, USA

\section{Authors contributions}

J.K.L. study concept and design, data interpretation, R.R. performed the in vitro functional assays, L.C. helped in genotyping and T.A. and Y.-C.W. performed statistical analysis; M.R.L, J.C., R.A., S.R., B.A.H., I.D., A.G.S., and S.M. designed, carried out the clinical trial, provided specimens and clinical data. All authors contributed to data interpretation, manuscript writing, and editing and critical revision.

\section{Conflict of interest}

M.R.L. is employee of Hemtologics Inc., I.D.B. has royalty from CD33 antibody used for leukemia diagnosis from Becton Dickinson; royalty for intellectual property on Notch-induced cord blood stem cell expansion. A.S.G. has consulting or advisory role with Pfizer, Novartis. R.A. has honoraria from SigmaTau Pharmaceuticals. The remaining authors declare that they have no conflict of interest.

\section{Publisher's note}

Springer Nature remains neutral with regard to jurisdictional claims in published maps and institutional affiliations.

Supplementary Information accompanies this paper at (https://doi.org/ 10.1038/s41408-019-0211-y).

Received: 27 February 2019 Accepted: 15 April 2019 Published online: 21 May 2019

\section{References}

1. Walter, R. B., Appelbaum, F. R., Estey, E. H. \& Bernstein, I. D. Acute myeloid leukemia stem cells and CD33-targeted immunotherapy. Blood 119, 6198-6208 (2012).

2. Burnett, A. K. et al. The addition of gemtuzumab ozogamicin to low-dose AraC improves remission rate but does not significantly prolong survival in older patients with acute myeloid leukaemia: results from the LRF AML14 and NCRI AML16 pick-a-winner comparison. Leukemia 27, 75-81 (2013).

3. Burnett, A. K. et al. Identification of patients with acute myeloblastic leukemia who benefit from the addition of gemtuzumab ozogamicin: results of the MRC AML15 trial. J. Clin. Oncol. 29, 369-377 (2011).

4. Burnett, A. K. et al. Addition of gemtuzumab ozogamicin to induction chemotherapy improves survival in older patients with acute myeloid leukemia. J. Clin. Oncol. 30, 3924-3931 (2012). 
5. Castaigne, S. et al. Effect of gemtuzumab ozogamicin on survival of adult patients with de-novo acute myeloid leukaemia (ALFA-0701): a randomised, open-label, phase 3 study. Lancet 379, 1508-1516 (2012).

6. Hills, R. K. et al. Addition of gemtuzumab ozogamicin to induction chemotherapy in adult patients with acute myeloid leukaemia: a meta-analysis of individual patient data from randomised controlled trials. Lancet Oncol. 15 986-996 (2014).

7. Cooper, T. M. et al. AAML03P1, a pilot study of the safety of gemtuzumab ozogamicin in combination with chemotherapy for newly diagnosed childhood acute myeloid leukemia: a report from the Children's Oncology Group. Cancer 118, 761-769 (2012).

8. Gamis, A. S. et al. Gemtuzumab ozogamicin in children and adolescents with de novo acute myeloid leukemia improves event-free survival by reducing relapse risk: results from the randomized phase III Children's Oncology Group trial AAML0531. J. Clin. Oncol. 32, 3021-3032 (2014).

9. Lamba, J. K. et al. CD33 splicing polymorphism determines gemtuzumab ozogamicin response in de novo acute myeloid leukemia: report from Randomized phase III Children's Oncology Group Trial AAML0531. J. Clin. Oncol. 35, 2674-2682 (2017)

10. Walter, R. B. et al. CD33 expression and P-glycoprotein-mediated drug efflux inversely correlate and predict clinical outcome in patients with acute myeloid leukemia treated with gemtuzumab ozogamicin monotherapy. Blood 109 4168-4170 (2007).

11. Morris, K. L., Adams, J. A. \& Liu, J. A. Effect of gemtuzumab ozogamicin on acute myeloid leukemia blast cells in vitro, as a single agent and combined with other cytotoxic cells. Br. J. Haematol. 135, 509-512 (2006).

12. Walter, R. B. et al. The peripheral benzodiazepine receptor ligand PK11195 overcomes different resistance mechanisms to sensitize AML cells to gemtuzumab ozogamicin. Blood 103, 4276-4284 (2004).

13. Walter, R. B. et al. Multidrug resistance protein attenuates gemtuzumab ozogamicin-induced cytotoxicity in acute myeloid leukemia cells. Blood 102, 1466-1473 (2003).
14. Linenberger, M. L. et al. Multidrug-resistance phenotype and clinical responses to gemtuzumab ozogamicin. Blood 98, 988-994 (2001).

15. Goemans, B. F. et al. Large interindividual differences in cellular sensitivity to calicheamicin may influence gemtuzumab ozogamicin response in acute myeloid leukemia. Leukemia 22, 2284-2285 (2008).

16. Pollard, J. A. et al. CD33 expression and its association with gemtuzumab ozogamicin response: results from the randomized phase III Children's Oncology Group Trial AAML0531. J. Clin. Oncol. 34, 747-755 (2016).

17. Pollard, J. A. et al. Correlation of CD33 expression level with disease characteristics and response to gemtuzumab ozogamicin containing chemotherapy in childhood AML. Blood 119, 3705-3711 (2012).

18. Wang, D., Johnson, A. D., Papp, A. C., Kroetz, D. L. \& Sadee, W. Multidrug resistance polypeptide 1 (MDR1, ABCB1) variant 3435C >T affects mRNA stability. Pharmacogenet. Genomics 15, 693-704 (2005).

19. Owen, A. et al. Relationship between the C3435T and G2677T(A) polymorphisms in the ABCB1 gene and P-glycoprotein expression in human liver. Br. J. Clin. Pharmacol. 59, 365-370 (2005).

20. Takane, H. et al. Haplotype-oriented genetic analysis and functional assessment of promoter variants in the MDR1 (ABCB1) gene. J. Pharmacol. Exp. Ther 311, 1179-1187 (2004).

21. Sauer, G. et al. Basal expression of the multidrug resistance gene 1 (MDR-1) is associated with the TT genotype at the polymorphic site C3435T in mammary and ovarian carcinoma cell lines. Cancer Lett. 185, 79-85 (2002).

22. Hitzl, M. et al. The C3435T mutation in the human MDR1 gene is associated with altered efflux of the P-glycoprotein substrate rhodamine 123 from CD56 +natural killer cells. Pharmacogenetics 11, 293-298 (2001).

23. Dey, S. Single nucleotide polymorphisms in human P-glycoprotein: its impact on drug delivery and disposition. Expert Opin. Drug Deliv. 3, 23-35 (2006).

24. Hoffmeyer, S. et al. Functional polymorphisms of the human multidrugresistance gene: multiple sequence variations and correlation of one allele with P-glycoprotein expression and activity in vivo. Proc. Natl Acad. Sci. USA 97, 3473-3478 (2000) 